\title{
ABBREVIATIONS
}

In spelling the names of Greek authors and their works, I have followed Liddell, Scott, and Jones Greek-English Lexicon (Oxford 1968); for Latin authors, the Oxford Latin Dictionary. For authors not in either of these works, and for abbreviations of modern reference works, I have for the most part followed the practice of the Oxford Classical Dictionary 2nd ed (Oxford 1970). In the text and notes, monographs and articles of special relevance to pumping technology have been cited by author and date, and the full titles can be found by consulting the bibliography. The abbreviations of periodical titles are those of L'Année philologique. The papyrological abbreviations (not listed below) are those suggested in J.F. Oates, R.S. Bagnall, and W.H. Willis Checklist of Editions of Greek Papyri and Ostraka 2nd ed: Bulletin of the American Society of Papyrologists supp 1 (1978). Dates are all AD unless marked BC. In addition to the abbreviations standard to English prose, the following abbreviations appear:

AA Archäologischer Anzeiger Berlin

ABSA Annual of the British School at Athens London

$A D$ Archaiologikón Deltion Athens

AEA Archivo Español de Arqueologia Madrid

AnnEp L'Année epigraphique Paris

AncSoc Ancient Society Louvain

ANRW Aufstieg und Niedergang der römischen Welt Berlin

ArchCam Archaeologia Cambrensis Cardiff

ARID Analecta Romana Instituti Danici Copenhagen

ASAE Annales du Service des Antiquités d’Égypte Cairo

$A W$ Antike Welt Zürich

BASP Bulletin of the American Society of Papyrologists New York 


\section{xiii Abbreviations}

BCAR Bullettino della Commissione Archeologica Comunale in Roma Rome BJ Bonner Jahrbücher Bonn

BJPES Bulletin of the Jewish Palestine Exploration Society

BMAH Bulletin des Musées royaux d Art et d Histoire Brussels

CAH Cambridge Ancient History

CahArchSub Cahiers d'Archéologie Subaquatique Fréjus

CE Chronique d'Égypte Brussels

CIL Corpus inscriptionum Latinarum

$\mathrm{CPh}$ Classical Philology Chicago

CQ Classical Quarterly Oxford

$C R$ Classical Review Oxford

CRAI Comptes rendus de l'Académie des Inscriptions de Belles-Lettres Paris Dar.-Sag. Ch. Daremberg and E. Saglio Dictionnaire des antiquités grecques et romaines $1877-1919$

EAA Enciclopedia dell arte antica, classica e orientale 1958-65

Frank Econ. Survey T. Frank ed An Economic Survey of Ancient Rome 5 vol, 1933-40

FGrH F. Jacoby Fragmente der griechischen Historiker 1923-

$G \& R$ Greece and Rome Oxford

GGA Göttingische Gelehrte Anzeigen Göttingen

HelbigA W. Helbig Führer durch die öffentlichen Sammlungen klassicher Altertümer in Rom 4th ed 1963-72

IEJ Israel Exploration Journal Jerusalem

IJNA International Journal of Nautical Archaeology London

ILN Illustrated London News London

ILS Dessau Inscriptiones Latinae selectae

JEA Journal of Egyptian Archaeology London

JFA Journal of Field Archaeology Boston

JHS Journal of Hellenic Studies London

JJP Journal of Juristic Papyrology Warsaw

Jones Documents ${ }^{2} \quad$ V. Ehrenberg and A.H.M. Jones Documents Illustrating

the Reigns of Augustus and Tiberius 2nd ed, Oxford 1955

JRS Journal of Roman Studies London

JSAH Journal of the Society of Architectural Historians Philadelphia

Liddell and Scott Liddell and Scott Greek-English Lexicon 9th ed, revised

by $\mathrm{H}$. Stuart Jones, Oxford 1925-40

MAAR Memoirs of the American Academy in Rome Rome

MAL Memorie della Classe di Scienze morali e storiche dell' Accademia dei

Lincei Rome

MCV Mélanges de la Casa de Velazquez, Madrid Paris

MDAI(K) Mitteilungen des Deutschen Archäologischen Instituts (Abt. Kairo) 


\section{xiv Abbreviations}

MDAI(M) Mitteilungen des Deutschen Archäologischen Instituts (Abt. Madrid)

Heidelberg

MDAI(R) Mitteilungen des Deutschen Archäologischen Instituts (Röm. Abt) Mainz

MEFR Mélanges d'Archéologie et d'histoire de l'École français de Rome Paris MonPitAnt Monumenti della Pittura Antica Rome

NSA Notizie degli Scavi di Antichità Rome

$O C D^{2}$ N.G.L. Hammond and H.H. Scullard The Oxford Classical Dictionary 2nd ed, Oxford 1970

PG Migne Patrologiae Cursus, series Graeca

Platner-Ashby S.B. Platner and T. Ashby A Topographical Dictionary of Ancient Rome Oxford 1929

RA Revue archéologique Paris

$R B i$ Revue biblique Paris

RE A. Pauly, G. Wissowa, and W. Kroll Real-Encyclopädie d. klassischen Altertumswissenschaft 1893-

REA Revue des études anciennes Talence

REG Revue des études grecques Paris

RPAA Rendiconti della Pontifica Accademia di Archeologia Rome

RPh Revue de philologie Paris

SIFC Studi Italiani di Filologia Classica Florence

SIG Dittenberger Sylloge inscriptionum Graecarum

Smith Dict W. Smith A Dictionary of Greek and Roman Antiquities 1901

$T \& C$ Technology and Culture Chicago

$T L G$ Stephanus Thesaurus linguae Graecae

$T L L$ Thesaurus linguae Latinae 